RESEARCH ETHICS

\title{
Non-therapeutic research with minors: how do chairpersons of German research ethics committees decide?
}

\author{
C Lenk, K Radenbach, M Dahl, C Wiesemann
}

See end of article for authors' affiliations

.....................

Correspondence to: Dr C Lenk, Department for Medical Ethics and History of Medicine,

Humboldtallee 36, 37073

Goettingen, Germany;

clenk@gwdg.de

Received 21 July 2003

Revised 1 October 2003

Accepted

20 November 2003

\begin{abstract}
Objectives: Clinical trials in humans in Germany-as in many other countries - must be approved by local research ethics committees (RECs). The current study has been designed to document and evaluate decisions of chairpersons of RECs in the problematic field of non-therapeutic research with minors. The authors' purpose was to examine whether non-therapeutic research was acceptable for chairpersons at all, and whether there was certainty on how to decide in research trials involving more than minimal risk. Design: In a questionnaire, REC chairpersons had to evaluate five different scenarios with (in parts) nontherapeutic research. The scenarios described realistic potential research projects with minors, involving increasing levels of risk for the research participants. The chairpersons had to decide whether the respective projects should be approved.

Methods: A total of 49 German REC chairpersons were sent questionnaires; 29 questionnaires were returned. The main measurements were approval or rejection of research scenarios.

Results: Chairpersons of German RECs generally tend to accept non-therapeutic research with minors if the apparent risk for the participating children is low. If the risk is clearly higher than "minimal", the chairpersons' decisions differ widely.

Conclusion: The fact that there seem to be different attitudes of chairpersons to non-therapeutic research with minors is problematic from an ethical point of view. It suggests a general uncertainty about the standards of protection for minor research participants in Germany. Therefore, further ethical and legal regulation of non-therapeutic research with minors in Germany seems necessary.
\end{abstract}

F or the last ten years in Germany, so called nontherapeutic research with study participants not competent to consent has been a target of passionate criticism. For example, in 1997, the "Eisingen case" caused a stir when a trainee of the St Josef's Home for the Handicapped in Bavaria blew the whistle on human genetic research with mentally handicapped inmates that had taken place without informed or proxy consent. A doctoral candidate from the Institute for Human Genetics of the University of Wuerzburg had taken blood samples from 179 residents without informing the people concerned or their parents or legal guardians. ${ }^{1}$ Criticism focussed not only on the lack of consent but also, and predominantly, on the alleged immorality of research without potential direct benefit.

The public reaction was fuelled by a debate in Germany on the European Convention on Human Rights and Biomedicine, in particular article 17 of the convention which deals with the "Protection of persons not able to consent to research". Human rights activists objected to the permission of minimal risk research with the non-competent. The Church and organisations for the disabled, in particular, warned against a deterioration of existing ethical and legal standards in Germany, should the convention be ratified. Moreover, for the same reason the revision of the Helsinki Declaration on Human Research (Edinburgh 2002), which allowed for this type of research, was received critically by German lawyers as well as the public. ${ }^{2}$ It is doubtful, however, whether Germany has reached high standards in the ethical and legal regulation of medical research. ${ }^{34}$

As in most industrialised countries, in Germany clinical trials involving humans must be approved by a local research ethics committee (REC). The relevant law on the approval of prescription drugs (Arzneimittelgesetz/AMG) demands that researchers ensure the safety of participants in clinical testing. The law regulates clinical testing according to basic standards in medical ethics: minimisation of risks for study participants, informed consent, and good clinical practice. In addition, clinical research with minors has to fulfil the following requirements: the drug in question is designed for the diagnosis or treatment of paediatric diseases, clinical trials with adults are not expected to yield adequate results, and parents (and if possible the child too) have to consent to study participation. However, the provisions in the AMG have been criticised for not being precise and detailed enough. It remains unclear, for example, whether the law allows for non-therapeutic research in minors or not (for a critical appraisal of this type of research see Nicholson ${ }^{5}$ and Brock $^{6}$ ).

Further regulations are to be found in a statement of the "Central Ethics Commission" (Zentrale Ethikkommission) at the German Federal Medical Council "On protecting noncompetent persons in medical research". ${ }^{7}$ According to these guidelines, minimal risk or burden in certain cases of nontherapeutic research may be acceptable even for vulnerable groups. Only if a vulnerable person shows significant unwillingness to participate in a study, has research to be stopped. However, the status of these recommendations is unclear, as they might be in conflict with existing laws. This situation creates a remarkable amount of uncertainty for the public, as well as for RECs.

The REC at Goettingen University, for example, in 2002 received a total of 13 research protocols dealing with minors. Eleven of the 13 protocols had elements of non-therapeutic research. So far, little is known about decisions German RECs actually make in the case of non-therapeutic research with minors: How do chairpersons decide in these cases? Are they willing to accept studies with no direct benefit for the

Abbreviations: REC, research ethics committee 


\section{Research project scenarios}

1 Using an additional $5 \mathrm{ml}$ blood from a blood sample primarily drawn for diagnostic/therapeutic reasons.

2 Various tests with healthy children: neurological examination, electroencephalogram, hearing test, questionnaires (duration: 7-8 hours over a period of 3 days).

3 Additional myocardial biopsy in the course of a heart operation performed for therapeutic purposes.

4 Additional bone marrow biopsies in leukaemia patients (6 out of 10 exclusively for non-therapeutic reasons).

5 Controlled clinical trial with toddlers, involving a placebo group which would have to undergo several intramuscular injections of sodium chloride solution.

participants? Which type of risk do they think is acceptable in research with minors?

In the present study, we do not intend to present an ethical solution for the problems involved. We are well aware of the fact that a normative problem cannot be tackled by analysing empirical data. However, we want to examine how those ultimately responsible for the ethical evaluation of research with minors in Germany decide, and whether their answers show ambiguity or certainty in decision making. We will also compare their answers with what are internationally perceived as acceptable risks. Our results will show whether German ethical standards in non-therapeutic research can in fact be judged to be comparatively high.

\section{STUDY DESIGN}

In order to answer the questions above, a research group at Goettingen University designed an empirical study to assess the decisions of chairpersons of German research ethics committees in cases of non-therapeutic research with minors. In accordance with Kopelman, ${ }^{8}$ by non-therapeutic research we understand study elements performed to seek "generalisable knowledge and not intended as therapy to benefit the individual directly". Forty nine REC chairpersons were contacted and asked to complete a questionnaire. In the questionnaire we asked for an evaluation of five realistic research scenarios, all examples of non-therapeutic research in paediatrics or child psychiatry with no direct benefit for the children concerned (see box). The risk and burden involved for the study participants gradually increased from scenario 1 to 5 (see box). We recorded 35 reactions $(71.4 \%)$; 29 questionnaires were filled out and sent back (59.2\%). Ninety per cent of those who answered were men, 51.7\%

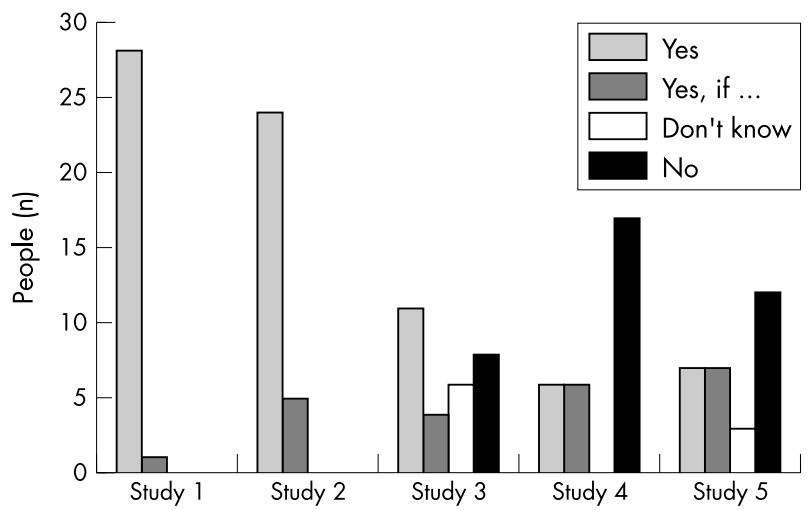

Figure 1 Evaluation of five different studies by chairpersons of German research ethics committees $(n=29)$. were older than 60 years, and 41.4\% were between 41 and 60 years old.

Eighty nine point seven per cent of the respondents were medical doctors, and $20.7 \%$ worked in the field of paediatrics. $72.4 \%$ of the respondents had extensive experience in the field of decision making in RECs-that is, they had taken part in more than 50 committee meetings; another $24.1 \%$ had participated in at least 10 to 50 meetings. Ninety three point one per cent of the respondents had children of their own.

We asked whether the research scenarios depicted would be evaluated positively by the chairperson ("Do you think the study is justifiable and would you support a positive decision of the research ethics committee?"). The respondents had four options: "Yes, without restrictions", "Yes, with restrictions (please explain)", "I don't know/I have no opinion", and "No, under no circumstances".

\section{RESULTS}

The answers of the respondents are shown in the figure. From the respondents' point of view, there was a significant difference between the appraisals of studies 1 and 2, and studies 3, 4, and 5. While research scenarios 1 and 2 would have been approved by all REC chairpersons (although sometimes with slight restrictions), trials 3 to 5 were strongly rejected by a number of participants. In our view, this division of opinion is caused by the far more invasive character of the latter research projects. While the study design of research projects 1 and 2 involved only a very small risk for the participants' health, the medical interventions in studies 3 to 5 were far riskier and could have caused severe side effects.

The design of study 1 had the highest acceptance (28/ $96.6 \%)$. It included the use of an additional $5 \mathrm{ml}$ blood from a blood sample for diagnostic reasons from children with a non-specific mental retardation. Although the study design as far as risks are concerned strongly resembled the German "Eisingen case" (see above), it seemed quite acceptable to chairpersons. The respondents could add comments to their evaluation of the respective research projects. Most of the comments on the evaluation of study l correctly referred to the necessity of obtaining the parents' or children's informed consent or assent.

The proposal for study 4 received the highest number of rejections. Children at the age of 6-11 suffering from acute lymphatic leukaemia were supposed to participate in a randomised, two armed study. Its purpose was to compare the outcome of a new chemotherapy with standard therapy. In the course of the study, weekly bone marrow biopsies were to be performed in both study groups. Six out of 10 bone marrow biopsies were intended for purely scientific purposes, without direct benefit for the participant involved. Most of the respondents (17/58.6\%) refused approval of the study. The most frequent comment on the study design was the rejection of additional, non-therapeutic bone marrow biopsies. Other frequent comments were critical of the high burden it put on the study participants, and of the treatment plan for the biopsies. One respondent referred to the additional biopsies as "a kind of child abuse". However, six chairpersons $(20.7 \%)$ saw no problem to approve the study without further restrictions.

Scenario 5 also elicited a wide range of reactions. The aim of the study was the treatment of children aged 2-5 with congenital cardiac defect. These patients have an increased risk of myocardial inflammation caused by respiratory syncytial viruses. The research programme proposed a randomised, two armed, placebo controlled study to prove the prophylactic effect of immunoglobin against heart muscle inflammation. The children in the placebo group were to receive intramuscular injections of sodium chloride solution. 
In the course of the study, cases of myocarditis in each group would be counted. Study participants with myocardial inflammation were to receive standard treatment. As the chart in the figure shows, the placebo controlled study (5) was more easily accepted by chairpersons than study 4 . Seven of the respondents $(24.1 \%)$ would have approved the study without further restrictions, while $12(41.4 \%)$ would have rejected it.

\section{CONCLUSION}

The results of our study show that in the case of nontherapeutic research with minors involving a higher than minimal risk, decisions of chairpersons of German RECs vary to a disturbingly high degree. In contrast to widespread public criticism, chairpersons do not object on principle to non-therapeutic research with only minimal risk or burden (for a critical discussion of the notion "minimal risk" see Kopelman ${ }^{9}$ and Maio $^{10}$ ). There seems to be a kind of consensus among chairpersons with regard to the evaluation of less invasive interventions for non-therapeutic research, as the undisputedly positive evaluation of studies 1 and 2 has shown. More invasive interventions like those involved in studies 3 to 5, however, are seen as more controversial. This reflects the ethical discussion about non-therapeutic research with minors in Germany. From our point of view, it is disturbing that RECs in Germany seem to arrive at widely differing decisions where more invasive interventions are concerned. Whereas four out of 29 committees, according to the vote of their chairpersons, would probably have refused the approval for at least three of the five studies, two committees might have approved all studies without any restrictions.

We believe this situation is not acceptable. We are aware of the fact that the opinion of chairpersons cannot simply be equated with the decision of the committees as such. However, chairpersons are usually experienced and well informed members of ethics committees as far as ethical and legal regulations are concerned. Their interpretation of what is legally and ethically acceptable on the basis of current German regulations should, where more than minor risks are at stake, at least not differ to such a wide extent. The European Convention on Human Rights and Biomedicine, for example, strictly rules out this type of research. That the opinions of German chairpersons on these issues vary to such a high degree reveals significant uncertainty as to the ethical standards in non-therapeutic research. This creates problems for study participants, researchers, and the public, who are left uncertain about the standards of protection of research participants and in risk benefit analyses. This problem is particularly prominent in multicentre research projects where several local RECs are involved. Our conclusion is that there is an urgent need for a more detailed, comprehensive, and unambiguous regulation of research with the non-competent in Germany that does not permit such a wide range of interpretation. Meanwhile, the ratification of the European Convention on Human Rights and Biomedicine seems to be a good way to guarantee at least minimal ethical standards in these cases in Germany.

\section{Authors' affiliations}

C Lenk*, K Radenbach*, M Dahl, C Wiesemann, Department for Medical Ethics and History of Medicine, University of Goettingen, Germany

*Both authors contributed equally to this article.

This study was funded by a research grant from the Volkswagen Vorab.

\section{REFERENCES}

1 Doerner K, Spielmann U, eds. Geistige Behinderung, Humangenetik und Ethik. Der Wuerzburg-Eisinger Fall. Eisingen: Verlag St Josefs-Stift Eisingen, 2001.

2 Taupitz J. Forschung am Menschen: Die neue Deklaration von Helsinki. Deutsches Ärzteblatt 2001;98(38): A-2413/B-2082/C-1933.

3 Wiesemann C. Die ethische Bewertung fremdnütziger Forschung in der Kinder- und Jugendmedizin. In: Wiesing U, Simon A, Engelhardt D, eds. Ethik in der medizinischen Forschung. Yearbook for Medical Ethics Vol 13, Stuttgart, Germany: Schattauer Verlag, 2000:71-81.

4 Wiesemann C, Dahl M. Forschung mit Kindern und Jugendlichen-Ist eine neue rechtliche Regelung notwendig? In: Wiesemann C, Doerries A, Simon A, Wolfslast G, eds. Das Kind als Patient. Ethische Konflikte zwischen Kindeswohl und Kindeswille. Frankfurt, Germany: Campus Verlag, 2003:264-280.

5 Nicholson R. The Ethics of Research with Children. In: Brazier M, Lobjoit M, eds. Protecting the Vulnerable. Autonomy and Consent in Health Care. London: Routledge, 1991:10-21.

6 Brock DW. Ethical Issues in Exposing Children to Risks in Research. In: Grodin MA, Glantz LH, eds. Children as Research Subjects: Science, Ethics, and Law. Oxford: Oxford University Press, 1994:81-101.

7 Zentrale Ethikkommission. Stellungnahme zum Schutz nichteinwilligungsfähiger Personen in der medizinischen Forschung. Deutsches Ärzteblatt 1997;94:A-1011.

8 Kopelman LM. Research Policy: Risk and Vulnerable Groups. In: Reich ThR, ed. Encyclopedia of Bioethics. Volume 4. Revised Edition. London: Simon \& Schuster and Prentice Hall International, 1995:2291-6.

9 Kopelman LM. When is the risk minimal enough for children to be research subjects? In: Kopelman LM, Moskop JC, eds. Children and Health Care: Moral and Social Issues. Dordrecht et al.: Kluwer Publishers, 1989:89-99.

10 Maio G. Zur Kritik der fremdnützigen Forschung an Kindern. Zeitschrift für medizinische Ethik 2001;47:173-87. 